\title{
A Coordination of Risk Management for Supply Chains Organized as Virtual Enterprises
}

\author{
Min Huang, ${ }^{1}$ Xingwei Wang, ${ }^{1}$ Fu-Qiang Lu, ${ }^{2}$ and Hua-Ling Bi ${ }^{2}$ \\ ${ }^{1}$ College of Information Science and Engineering, Northeastern University, State Key Laboratory of Integrated Automation of \\ Process Industries, Shenyang, Liaoning 110819, China \\ ${ }^{2}$ Northeastern University at Qinhuangdao, Qinhuangdao 066004, China
}

Correspondence should be addressed to Fu-Qiang Lu; fuqiang_lu@126.com

Received 21 July 2012; Accepted 6 January 2013

Academic Editor: Xiaohang Yue

Copyright (C) 2013 Min Huang et al. This is an open access article distributed under the Creative Commons Attribution License, which permits unrestricted use, distribution, and reproduction in any medium, provided the original work is properly cited.

\begin{abstract}
As a new management mode, great attention has been paid to virtual enterprise (VE). While there is much research material on risk management of $\mathrm{VE}$, a relationship perspective on owner and partner performance assessment and management can bring an added dimension. The coordination of risk management in fashion and textiles (FTs) supply chain organized as a VE is studied in this paper. The aim of this study is to find proper decision mechanisms that can improve the overall performance of risk management for the whole VE as well as each member. For the risk management problem in VE, a centralized mechanism is given as the base case, and then a distributed decision-making (DDM) mechanism with incentive scheme is introduced to establish a practicable strategic partnership. Under the DDM mechanism, a relationship performance definition that incorporates the financial dimension is investigated. For the two resulting optimization problems, a particle swarm optimization (PSO) algorithm is designed. In the numerical examples, the study shows that the DDM mechanism with incentive scheme can improve the overall benefit of risk management beyond the centralized one. Additionally, sensitivity analysis is conducted with respect to the bonus parameter, and suggestions are made for further research.
\end{abstract}

\section{Introduction}

In the past decade, fashion and textiles (FTs) industry become one of the most rapidly developed industries. Virtual enterprise (VE) is becoming the important organized pattern of FTs supply chain. VE is defined as an organization that brings complementary individuals and interest groups together in an association in order to meet short-term objectives and exploit fast changing market trends. The essential characteristics of VE are as follows: the involvement of legally independent partners, a limited lifespan, the existence of a common mission for all participants, a focus on each partner's key competencies, and the intensive use of information technologies [1]. The virtual nature of this kind of organization results from the fact that these enterprises do not really have any actual physical existence but exist through the pooling of resources belonging to each of the partners. Typical examples of virtual enterprises are Amazon.com, which was originally a bookstore that sold exclusively on the Internet and now retails a multitude of products, and Dell Computer, the world's leading computer manufacturer. As a VE is a complex system temporarily composed of many stand-alone enterprises due to market opportunities, many factors, such as changing of price and demand, insufficient knowledge sharing, lack of cooperation among members, and weaknesses in resources, can cause risks [2]. Hence, risk management is the key problem to overcome in a VE to ensure success.

In the past, various models and mechanisms have been developed to provide a more scientific and effective way for managing the risk of a VE. Zhi (1995) [3] proposes a risk assessment method which combines the risk probability analysis with the risk impact assessment. The method incorporates various sources of risks attributed to overseas construction projects. Das and Teng (1998) [4] suggest two basic types of risk in strategic alliances: relational risk and performance risk. The alliance-making process is examined in terms of the interactive effects of resource and risk on the orientations and objectives of the prospective alliance partners. This type 
of alliance is similar to the VE. Grabowski and Roberts (1999) [5] identify four processes which are important to risk mitigation in virtual organizations. These processes are organizational structure and design, communication, culture, and trust. They then suggest how these processes may enhance the reliability of the virtual organizations and discuss how thoughtful the management of those attributes can mitigate the risk. Ip et al. (2003) [6] consider minimizing the risk in selecting partners and ensuring the due date of a project in a VE. They propose a risk-based partner selection model. By exploring the characteristics of the problem considered and the knowledge of project scheduling, a rule-based genetic algorithm (R-GA) with embedded project scheduling is developed to solve the problem. Karjalainen et al. (2004) [7] use a case study approach to explore the implementation of profit and risk-sharing mechanisms in a VE. Lack of a shared vision may have been the most important cause for the early decomposition of the VE. Therefore, the trust did not start to accumulate during the cooperation. This would have been imperative for the implementation of profit sharing mechanisms, because risk attitudes seemed to favor hierarchical rewarding mechanisms. Huang et al. (2008) [8] focuse on two main features of the $\mathrm{VE}$, project mode and uncertain factors. They establish the fuzzy synthetic evaluation embedded nonlinear integer programming model of risk programming for the VE and present a tabu search algorithm with an embedded fuzzy synthetic evaluation for the model. Wei et al. (2008) [9] introduce the theory of fuzzy cognitive time maps (FCTMs) into modeling and evaluating trust relationships and show how relevant the inter-organizational trust is based on trust sources and their credibility. They also propose a methodology by taking dynamic nature of trust into account to analyze the evolution of trust in the VEs setting. The established cognitive map illustrates the changes of different factors and their effects on the final trusts. Chen et al. (2010) [10] design a trust-based knowledgesharing model based on characteristics of VEs and the knowledge structure model to express knowledge associated with VE activities. The proposed knowledge-sharing method provides the trust level between a knowledge-requesting enterprise and a knowledge-supplying enterprise to improve the willingness of the latter to share more valuable knowledge, ultimately increasing the efficiency and competitiveness of VEs. Huang et al. (2011) [11] develop a distributed decisionmaking (DDM) model for risk management of the VE. The model has two levels, namely, the top model and the base model, which describe the decision processes of the owner and the partners of the VE, respectively. A particle swarm optimization (PSO) algorithm is then designed to solve the resulting optimization problem. The result shows that the proposed algorithm is effective and the two-level model can help to improve the description of the relationship between the owner and the partners, which is helpful to reduce the risk of the VE.

Much of the existing literature focuses on identifying risk, providing risk evaluation method, and developing risk management models. Many models proposed in the literature only discuss the risk management issues in a traditional centralized structure, few develop distributed decision-making model by considering the diversity of the members and the distribution characteristics of the VE. But the models they proposed did not involve any additional way, like incentives, to improve the coordination of members in VE.

This study attempts to establish the risk management of VE by determining appropriate mechanisms of coordination and independency among members in the VE. The study differs from the existing literature in that it not only attempts to discuss the risk management issues in a functional centralized mechanism, but also investigates distributed decisionmaking ones in terms of financial incentives which, in addition to giving a higher level of coordination among all parties in the VE, maximizes the benefit of the whole VE. In view of the fact that the models of the risk management problem are considered as NP-hard, and a nonlinear with discrete variables, a particle swarm optimization (PSO) is designed. The comparative study between the distributed decision-making (DDM) mechanism with incentive schemes and the centralized mechanism is presented in the numerical examples.

The rest of this paper is structured as follows. In Section 2, the problem of risk management in the VE is described. Assumptions and notations, centralized model, and the DDM model with incentives are given in Section 3. A PSO algorithm is designed for the resulting optimization problem, which is shown in Section 4. Numerical examples are presented in Section 5. Concluding remarks and further research are given in the final section.

\section{Problem Formulation of Risk Management}

2.1. Problem Description. The risk management problem can be described as follows.

In an FTs supply chain organized as VE, the owner (an FTs enterprise) finds a business project consisting of several subprojects. The owner is not able to complete the whole project using its own capacity. Therefore, it has to invite partners for the subprojects. Of course, the risk management of the sub-projects is also an important responsibility of partners. The owner determines the upper bound of the budget for each subproject first. The partners who accept the budget condition will respond to the risk management of its sub-project and propose the benefit of the risk management that they need to finish according to the resources they have. The risk loss for subproject should not go beyond the value which is expected by the owner. In this process, the owner manages the risk of the entire project to ensure the success of $\mathrm{VE}$, while maximizing the benefit of risk management from the entire project.

For each partner, there are several risk factors which threaten its safety. Being dealt with risk control strategies, the risk factor will be controlled by some way. There are some strategies for each risk factor. The effect of each strategy on the corresponding risk is different. Thus, the description for each risk factor and the cost of the different control strategies are different. The partner has to maximize its benefit of risk management by optimally combining these strategies, under the budget allocated by the owner. The benefit of 


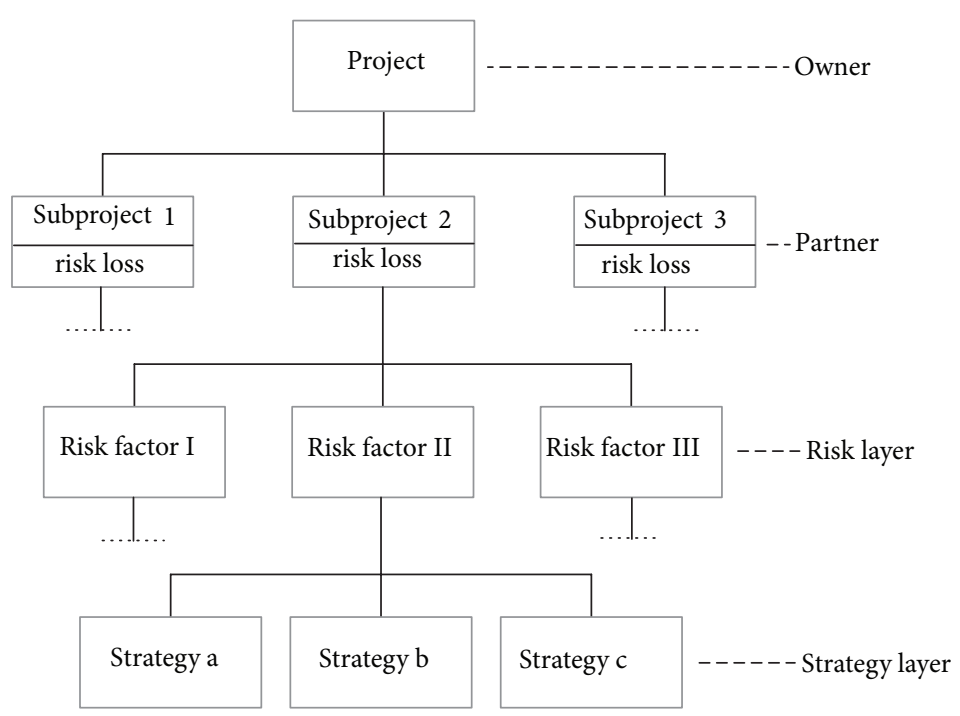

FIgURE 1: The conceptual representation of risk management in VE.

risk management for each partner/subproject is a difference between the initial risk loss of the subproject and the risk loss under risk management. The conceptual representation of risk management in VE is shown in Figure 1; here a business project consisting of three sub-projects is taken as an example.

2.2. Notations and Assumptions. For comparison purposes, the models in this study are constructed and separated according to the centralized mechanism and the DDM mechanism respectively, which are the centralized model and improved DDM model with incentives.

The following notations and assumptions are used in all the studied models.

\section{Notations}

(i) $B_{i}$ : upper bound of the budget for partner $i, i=$ $1,2, \ldots, M,(\$)$.

(ii) $B_{\max }$ : upper bound of the total budget for risk management of the VE (\$).

(iii) Bonus $s_{i}$ an incentive that the owner pays to the partner when the risk loss of partner $i$ down to the target value of the owner (\$).

(iv) $\operatorname{Benefit}_{i}\left(x_{i}\right)$ : benefit of risk management for partner $i$ under budget $x_{i}(\$)$.

(v) bonus: : bonus for partner $i(\$)$.

(vi) $C_{i j}\left(y_{i j}\right)$ : cost of risk control strategy $y_{i j}(\$), i=$ $1,2, \ldots, M, j=1,2, \ldots, N_{i}$.

(vii) cost $_{\mathrm{ac}}$ : activated cost for each partner (\$).

(viii) $\mathrm{ER}_{i}$ : target value of risk loss of partner $i$, required by the owner (\$).

(ix) $\mathrm{EC}_{i}$ : cost to activate the target value of risk loss for partner $i(\$)$.

(x) $L_{0}^{\text {initial }}$ : initial state of risk loss for owner (\$). (xi) $L_{i}^{\text {initial }}$ : initial state of risk loss for partner $i$ in the VE (\$).

(xii) $L_{i}\left(x_{i}, \mathrm{EC}_{i}\right)$ : risk loss of Partner $i$ under budget $x_{i}$ and the activated cost $\mathrm{EC}_{i}$.

(xiii) $L_{0}\left(x_{0}\right)$ : risk loss of owner under budget $x_{0}(\$)$.

(xiv) $L_{i}\left(x_{i}\right)$ : risk loss of Partner $i$ under budget $x_{i}$, and $L_{i}\left(x_{i}\right)=p_{i j} l_{i j}\left(y_{i j}\right)$.

(xv) $l_{i j}\left(y_{i j}\right)$ : risk loss from risk factor $j$ of partner $i$ under strategy $y_{i j}(\$)$.

(xvi) $M$ : number of partners in the VE.

(xvii) $N_{i}$ : number of risk factor for partner $i$.

(xviii) $p_{i j}$ : probability of risk occurrence for risk factor $j$ of partner $i$.

(xix) $W_{i j}$ : number of available strategy for risk factor $j$ of partner $i$.

(xx) $x_{0}$ : budget for owner.

(xxi) $x_{i}$ : budget for Partner $i$.

(xxii) $y_{i j}$ : risk control strategy selected for risk factor $j$ of partner $i$.

\section{Assumptions}

(1) The VE in the study consists of one owner and several partners.

(2) One partner responds to one subproject.

(3) It is assumed that the failure of risk management for each sub-project is an independent event.

(4) The impact of different risk factors on one subproject/partner is assumed to be an independent event.

(5) Generally the bonus for the partner is not-less than its activated cost. 
(6) Only one strategy will be selected for each risk factor or will do nothing with it.

(7) All information referred to the risk management process is available for the owner, such as the initial risk loss and the risk loss under the allocated budget of each partner.

(8) The value of activated cost for each partner is the same.

2.3. The Centralized Model. The centralized model views the system as one entity where there is one central planner (owner) who makes all decisions so as to maximize the benefit from the risk management of the whole system. Under this situation, a casual relationship is formed between owner and partner, but there is no mechanism to push forward their mutual trust. Each party must still be aware of the uncertainty caused by the other, such as whether the budget will be allocated correctly and on time to the partner or whether a strong enough risk control strategy will be selected to mitigate the risk by the partner.

\section{The centralized model:}

$$
\begin{aligned}
\max & {\left[L_{0}^{\text {initial }}-L_{0}\left(x_{0}\right)-x_{0}\right] } \\
& +\sum_{i=1}^{M}\left[L_{i}^{\text {initial }}-\sum_{j=1}^{N_{i}} p_{i j} l_{i j}\left(y_{i j}\right)-x_{i}\right]
\end{aligned}
$$

subject to

$$
\begin{aligned}
& \sum_{i=0}^{M} x_{i} \leq B_{\max }, \\
& \sum_{j=1}^{N_{i}} C_{i j}\left(y_{i j}\right) \leq x_{i}, \\
& x_{i} \in\left[0, B_{i}\right], \quad i=0,1, \ldots, M, \\
& y_{i j} \in\left\{0,1, \ldots, W_{i j}\right\}, \quad i=1, \ldots, M, j=0,1, \ldots, N_{i} .
\end{aligned}
$$

The goal of risk management in the study is to maximize the benefit of risk management, which consists of the benefit of risk management from owner and partners. The benefit is a difference between the downsize of risk loss and the allocated budget, as shown in (1). In (2), the total budget for risk management should not go beyond the the upper bound. The cost of risk management on sub-project should be not go beyond its allocated budget; see (3). Equations (4) and (5) are the interval and set of decision variables $x_{i}$ and $y_{i j}$, respectively.

2.4. The DDM Model with Incentives. VE involves the coordination of independently managed enterprises/partners who seek to maximize their own profits. Although overall performance of the VE on the partners' joint performance, the operational goals may conflict and result in an inefficiency for risk management of the entire VE. Therefore, one of the main

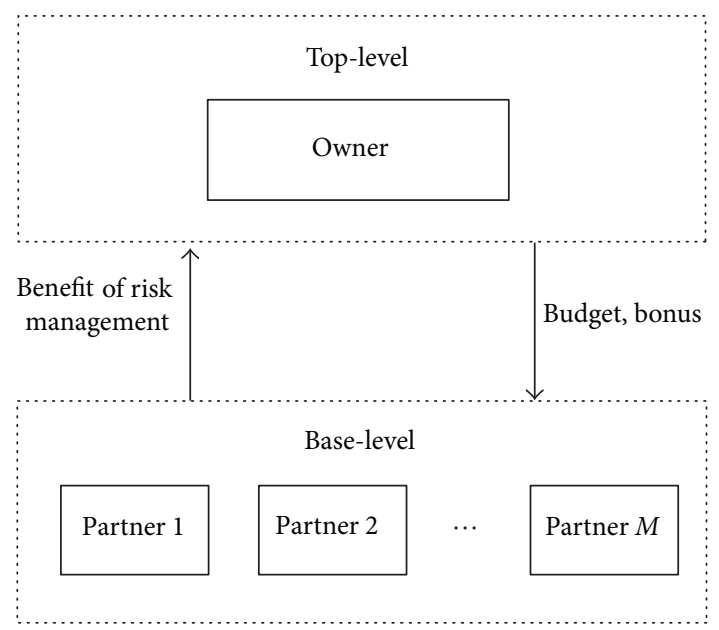

FIGURE 2: The DDM model for risk management of VE.

issues in risk management of VE is to find suitable decision mechanisms for coordinating the risk management processes that are controlled by owner and various partners, in order to achieve an overall maximum benefit from risk management $[12,13]$.

A DDM model with incentives is proposed to enhance the relationship formed between owner and partners. In practice, mutual relationship and trustful information are hardly achieved for independent companies without any financial benefits. This should be the first step leading to the full cooperation from members in the VE. Therefore, the owner should contribute some incentives to partners as a means to make a link between them and as an aim to improve the performance of the $\mathrm{VE}$ as well as their own company. The decision process of risk management is described by a two-level DDM model, namely, top-level and base-level, which demonstrates the decision process of owner and partners, respectively [14-16]. The DDM model is depicted in Figure 2, here the owner offers budget and bonus (the incentive) to partners, and the partner returns the benefit of risk management as a response.

The DDM Model. The top-level:

$$
\begin{aligned}
\max & {\left[L_{0}^{\text {initial }}-L_{0}\left(x_{0}\right)-x_{0}\right] } \\
& +\sum_{i=1}^{M}\left[L_{i}^{\text {initial }}-L_{i}\left(x_{i}\right)-x_{i}-\text { Bonus }_{i}\right]
\end{aligned}
$$

subject to

$$
\begin{aligned}
& \sum_{i=0}^{M} x_{i} \leq B_{\max } \\
& \text { Bonus }_{i}= \begin{cases}\text { Bonus }_{i} & \text { if } L_{i}\left(x_{i}\right) \leq \mathrm{ER}_{i}, \quad i=1,2, \ldots, M \\
0 & \text { otherwise }\end{cases} \\
& x_{i} \in\left[0, B_{i}\right] \quad i=0,1, \ldots, M
\end{aligned}
$$


In top-level, the decision maker is the owner who allocates the budget to the members of the VE, including himself/herself. The owner's aim is to maximize the benefit of risk management for the whole VE; the decision variables are therefore given by $\left(x_{0}, x_{1}, \ldots, x_{M}\right)$. A bonus is used as an incentive, which will be payed to partners when the risk loss of the partner is not beyond the target value of the owner, see (8). Equation (7) presents the constraint of total budget, and (9) gives the interval of the decision variables.

The base-level:

$$
\begin{aligned}
& \max \operatorname{Benefit}_{i}\left(x_{i} \text { Bonus }_{i}\right) \\
& =L_{i}^{\text {initial }}-\sum_{j=1}^{N_{i}} p_{i j} l_{i j}\left(y_{i j}\right)+\text { Bonus }_{i}-\mathrm{EC}_{i} .
\end{aligned}
$$

Subject to

$$
\begin{aligned}
& \sum_{j=1}^{N_{i}} C_{i j}\left(y_{i j}\right) \leq x_{i}+\mathrm{EC}_{i}, \\
& \mathrm{EC}_{i}=\left\{\begin{array}{cc}
\operatorname{cost}_{\mathrm{ac}} & \text { if } \sum_{j=1}^{N_{i}} p_{i j} l_{i j}\left(y_{i j}\right)>\mathrm{ER}_{i}, \\
& L_{i}\left(x_{i}, \mathrm{EC}_{i}\right) \leq \mathrm{ER}_{i}, \\
0 & \text { otherwise, }
\end{array}\right. \\
& \operatorname{cost}_{\mathrm{ac}}=\gamma \text { Bonus }_{i} \text {, } \\
& y_{i j} \in\left\{0,1, \ldots, W_{i j}\right\} \quad j=1, \ldots, N_{j} .
\end{aligned}
$$

In base-level, the decision makers are the partners and there are $M$ partners in base-level. Take the model for partner $i$ as an example. Partner $i$ selects the optimal combination of risk control strategies to maximize its benefit of risk management under the allocated budget and bonus, which is calculated by (10). However, to get the bonus, the partner also needs to prepare himself/herself for reducing its risk loss and meets the owner's requirements. From this perspective, the partner has an option to pay an extra cost (activated cost) to avoid the falling, reducing its risk loss. While the current risk loss of partner $i$ does not meet the target value of the owner and the risk loss of partner $i$ under the budget and its extra effort can meet the target value of the owner, the partner will pay the activated cost to reduce its risk loss; see (12). In this situation, the budget has increment for the partner, which is shown in (11). In (13), $\gamma$ is an activated cost parameter, $\gamma \in$ $(0,1)$, which means that the effort or the extra cost of partner $i$ has a direct proportion with the bonus. So, a bigger bonus will stimulate a greater effort. The set of decision variables $y_{i j}$ is presented in (14).

2.5. Decision Variables. There are two decision variables in the model, which are the budget for members $\left(x_{i}\right)$ and the risk control strategies selected for the risk factors of partners $\left(y_{i j}\right)$. The first variable $\left(x_{i}\right)$ is a continuous one, and the second variable $\left(y_{i j}\right)$ is a discrete one.

\section{Particle Swarm Optimization}

Due to the complexity of the studied models, which include a number of if-else conditions (as seen in Section 2), the problem in this study is considered as NP-hard and nonlinear with discrete variables, which is renowned for its difficulty in solving, particularly if optimal solutions are required. The two principal methods used to solve NP-hard problems are based on the enumeration method and artificial intelligence (AI). Enumeration method guarantees optimal solutions, but it is difficult to be used in some applications due to the fact that its performance does not scale well on larger problems. As a consequence, it has been enhanced with the addition of heuristics. Among various AI methods, the particle swarm optimization (PSO) has been widely used in recent years by many researchers to overcome the drawbacks of the mathematical models [17]. Therefore, PSO is selected to solve the problem in this study. The PSO was proposed by Kennedy and Eberhart in 1995, which is an optimization technique inspired by social behavior observable in nature $[18,19]$.

To simplify the discussion, let $W_{i j}=W$ and $N_{i}=N$, hence $i=1,2, \ldots, M, j=1,2, \ldots, N$. In the following parts, the main features of PSO will be outlined.

3.1. Particle Representation. For the continuous variable $x_{i}$, the real number in the range $\left[0, B_{i}\right], i=0,1, \ldots, M$ is selected as a particle. Each particle represents an allocation of budget among members of VE. The representation scheme of the $k$ th particle $\mathbf{X}_{k}=\left(x_{k 0}, x_{k 1}, \ldots, x_{k M}\right), k=1,2, \ldots, N_{\mathrm{PC}} \cdot N_{\mathrm{PC}}$ is the population size of the swarm for the continuous problem, and the population is named continuous-swarm.

For the discrete variable $y_{i j}$, the particle is composed of the binary number. Each particle represents a risk control strategies combination of its corresponding partner. A particle is a binary matrix; take the $t$ th one for example,

$$
\mathbf{Y}_{t}=\left(\begin{array}{cccc}
y_{t 11} & y_{t 12} & \cdots & y_{t 1 W} \\
y_{t 21} & y_{t 22} & \cdots & y_{t 2 W} \\
\vdots & \vdots & \vdots & \vdots \\
Y_{t N 1} & y_{t N 2} & \cdots & y_{t N W}
\end{array}\right), \quad t=1, \ldots, N_{\mathrm{PD}}
$$

The row of the matrix stands for risk factors and the column stands for risk control strategies. Element $y_{\text {th }}$ is a binary number, $t=1, \ldots, N_{\mathrm{PD}}, l=1,2, \ldots, N$, and $h=$ $1,2, \ldots, W . N_{\mathrm{PD}}$ is the population size of the swarm for the discrete problem, the population is named discrete-swarm, for example, a risk control strategies combination (2104); the representation scheme is

$$
\left(\begin{array}{llll}
0 & 1 & 0 & 0 \\
1 & 0 & 0 & 0 \\
0 & 0 & 0 & 0 \\
0 & 0 & 0 & 1
\end{array}\right)
$$

Here " 1 " denotes that the strategy is selected for its corresponding risk factor. "0" denotes that no strategy has been selected for the corresponding risk factor. 
3.2. Fitness Functions. In the two types of models, the fitness of particles is calculated by the following different fitness functions.

The fitness function for the centralized model:

$$
\begin{aligned}
F_{C}= & {\left[L_{0}^{\text {initial }}-L_{0}\left(x_{0}\right)-x_{0}\right] } \\
& +\sum_{i=1}^{M}\left[L_{i}^{i \text { nitial }}-\sum_{j=1}^{N} p_{i j} l_{i j}\left(y_{i j}\right)-x_{i}\right] \\
& -\alpha\left(\sum_{i=0}^{M} x_{i}-B_{\max }\right)^{+}-\beta\left(\sum_{j=1}^{N} C_{i j}\left(y_{i j}\right)-x_{i}\right)^{+} .
\end{aligned}
$$

Here $F_{C}$ is the fitness of particles for the centralized model. $\alpha$ and $\beta$ are punishment coefficients. Here notation $(\cdot)^{+}$is defined as below,

$$
(x)^{+}= \begin{cases}x & \text { if } x>0 \\ 0 & \text { otherwise }\end{cases}
$$

According to the two-level structure of the DDM model, the fitness functions for the top-level and the base-level are presented, respectively.

The fitness function for the top-level:

$$
\begin{aligned}
F_{T}= & {\left[L_{0}^{\text {initial }}-L_{0}\left(x_{0}\right)-x_{0}\right] } \\
& +\sum_{i=1}^{M}\left[L_{i}^{\text {initial }}-L_{i}\left(x_{i}\right)-x_{i}-\text { Bonus }_{i}\right] \\
& -\alpha\left(\sum_{i=0}^{M} x_{i}-B_{\max }\right)^{+} .
\end{aligned}
$$

Here $F_{T}$ is the fitness of particles for the top-level; $\alpha$ is the punishment coefficient.

The fitness function for the base-level:

$$
\begin{aligned}
F_{B}= & L_{i}^{\text {initial }}-\sum_{j=1}^{N_{i}} p_{i j} l_{i j}\left(y_{i j}\right)+\text { Bonus }_{i}-\mathrm{EC}_{i} \\
& -\beta\left(\sum_{j=1}^{N_{i}} C_{i j}\left(y_{i j}\right)-x_{i}-\mathrm{EC}_{i}\right)^{+} .
\end{aligned}
$$

Here $F_{B}$ is the fitness of particles for the base-level; $\beta$ is the punishment coefficient.

3.3. Updating of Particles. The particles are updated by different formulas for the continuous and discrete problems respectively.

\section{The continuous problem:}

$$
\begin{gathered}
v_{k i}=r\left(v_{k i}+c_{1} r_{1}\left(p_{k i}-x_{k i}\right)+c_{2} r_{2}\left(p_{g i}-x_{k i}\right)\right), \\
x_{k i}=x_{k i}+v_{k i} .
\end{gathered}
$$

Here $v_{k i}$ and $x_{k i}$ are the velocity and position of particle $k$, respectively, $v_{k i} \in\left\{-B_{i}, B_{i}\right\} . p_{k i}$ is the best position of particle $k$, among its previous positions. $p_{g i}$ is the best position found so far of the continuous-swarm. $r$ is the constriction factor, which is usually fixed $r=0.729 . r_{1}$ and $r_{2}$ are random values in the range $[0,1] . c_{1}$ and $c_{2}$ are acceleration constants, which control how far a particle will move in a single iteration. The values $r_{1} c_{1}$ and $r_{2} c_{2}$ determine the weights of cognition part and social part, respectively, where their sum is usually limited to generally, $c_{1} r_{1}+c_{2} r_{2}=4$ [20-23].

The discrete problem:

$$
v_{t l h}=r v_{t l h}+r_{1} c_{1}\left(p_{t l}-x_{t l h}\right)+r_{2} c_{2}\left(p_{g l}-x_{t l h}\right),
$$

where $v_{t l h}$ and $x_{t l h}$ are the velocity and position of particle $t$ in row $l$ and column $h$, respectively. $p_{t l}$ is the best position of particle $t$ among its previous positions in dimension $l . p_{g l}$ is the best position found so far in dimension $l$ within discreteswarm. $r$ is the inertia factor. $r_{1}$ and $r_{2}$ are random values, where $r_{1}, r_{2} \in[0,1] ; c_{1}$ and $c_{2}$ are acceleration constants, their sum is usually limited to 4 [20-23].

The velocity value can be constrained in the interval $[0,1]$ by using the following sigmoid function [19-21]:

$$
s\left(v_{t l h}\right)=\frac{1}{1+\exp \left(-v_{t l h}\right)} .
$$

$s\left(v_{t l h}\right)$ denotes the probability of element $x_{t l h}$ taking 1 . To avoid $s\left(v_{t l h}\right)$ approaching 0 or 1 , a constraint is used to limit the range of $v_{t l h}$; here $v_{t l h} \in[-W, W]$.

For this problem, only one strategy will be selected for one risk factor or will do nothing with it. Considering this characteristic, in particle $t$, strategy $h$ is selected for risk factor $l$ according to following probability:

$$
p_{t}(l, h)=\frac{s\left(v_{t l h}\right)}{\sum_{h}^{W} s\left(v_{t l h}\right)} .
$$

For particle $\mathbf{X}_{t}$, the position is updated by a designed process according to the characteristic of the problem (see Algorithm 1).

3.4. Termination Rule. The maximum number of iteration is used as the termination rule. For the centralized problem, the maximum number of iteration is $N_{\text {IC }}$. For the two-level structure of the DDM problem, the maximum number of iteration for the top-level is $N_{\mathrm{IT}}$. Correspondingly, $N_{\mathrm{IB}}$ is the maximum number of iteration for the base-level.

3.5. The Procedure of PSO. The procedure of PSO for the centralized problem is shown in Figure 3.

The procedure of PSO for the DDM problem is shown in Figure 4 .

\section{Numerical Examples and Insights}

4.1. An Illustrative Example. An example of an FTs supply chain organized as VE is built to demonstrate the proposed 


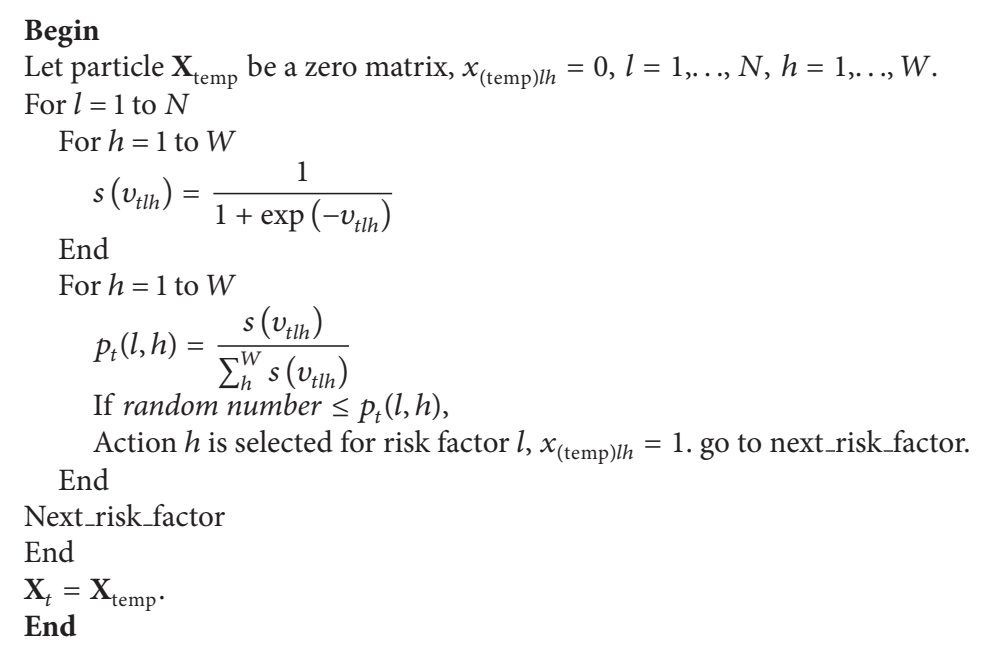

Algorithm 1

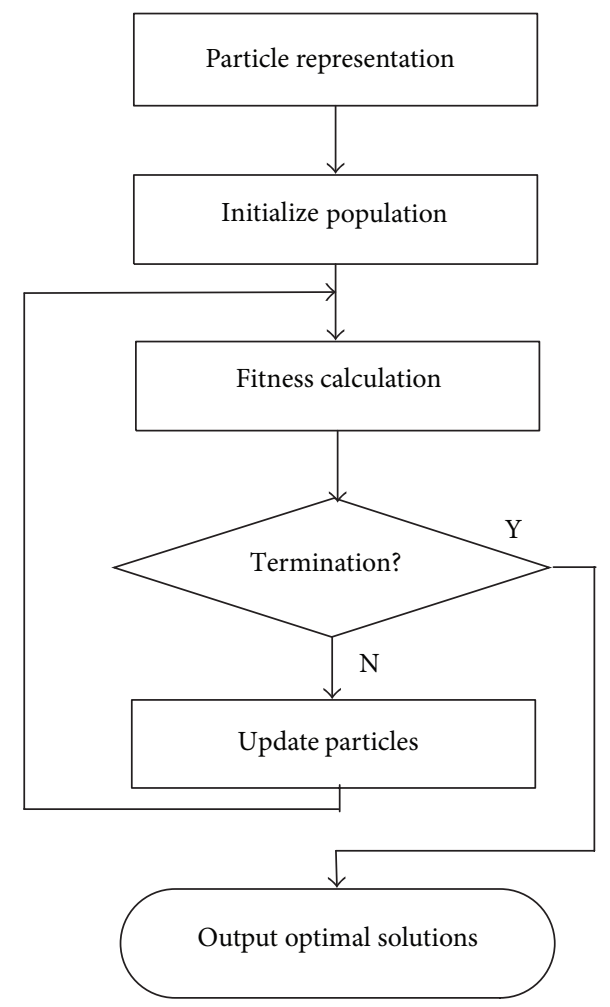

FIGURE 3: The procedures of PSO for the centralized problem.

algorithm and highlight the financial benefits from the incentive. One owner and one partner are considered in the example, so the number of members in the VE is $2, M+1=2$. Firstly, the owner has to allocate the budget to the partner and himself/herself, and the upper bound of the total budget is $\$ 1,200, B_{\max }=1,200$. The upper bound of the budget for owner and partner is $\$ 1,200$, respectively, $B_{i}=1200, i=1,2$.
The risk loss of the owner is up to his/her allocated budget, which is given by a convex decreasing function:

$$
L_{0}\left(x_{0}\right)=3000 \exp \left(-0.002 x_{0}\right) .
$$

For the partner, there are $10(N=10)$ risk factors which have the potential to threaten its safety, and $4(W=$ 4) risk control strategies are provided for each risk factor. In this example, only one of the strategies will be selected for each risk factor or will do nothing with it. The strategies are sequenced from low to high ac-cording to their effect. Generally, a stronger effect corresponds to a bigger index of the strategy. The probability of risk occurrence for each risk factor is $p_{i j}=\{0.97,0.90,0.77,0.70,0.57$, $0.47,0.30,0.22,0.20,0.07\}, i=1, j=1,2, \ldots, N$. The risk loss function is a convex decreasing function:

$$
l_{i j}\left(y_{i j}\right)=w_{i j} \exp \left(-\chi_{i j} y_{1 j}\right)
$$

The parameters $w_{i j}=\{100,200,300,400,500,700,900$, $1800,2200,7000\}, i=1, j=1,2, \ldots, N$, which are set according to the values of the probability of risk occurrence. The parameter $\chi_{i j}$ is used to describe the effect of various risk factors on risk loss; the values of the parameters are $\chi_{i j}=\{0.94,0.87,0.83,0.73,0.63,0.50,0.37,0.33,0.23,0.1\}$, $i=1, j=1,2, \ldots, N$. According to the specific form of risk loss function and the probability of risk occurrence, the initial risk loss for the owner and the partner is both $\$ 3,000, L_{i}^{\text {initial }}=3000, i=0,1$. The total initial risk loss of $\mathrm{VE}$ is $\$ 6,000$.

The partner has to pay for the cost of risk control strategies under its budget. The cost of the strategy is assumed to be a concave increasing function of the corresponding strategy, which is approximated by

$$
C_{i j}\left(y_{i j}\right)=300\left[1-\exp \left(-\epsilon_{i j} y_{i j}\right)\right], \quad i=1 .
$$

Here the parameter $\epsilon_{i j}$ is set according to the risk factors, $\epsilon_{i j}=\{0.1,0.2,0.3,0.4,0.5,0.6,0.7,0.8,0.9,1\}, j=1,2, \ldots, N$. 


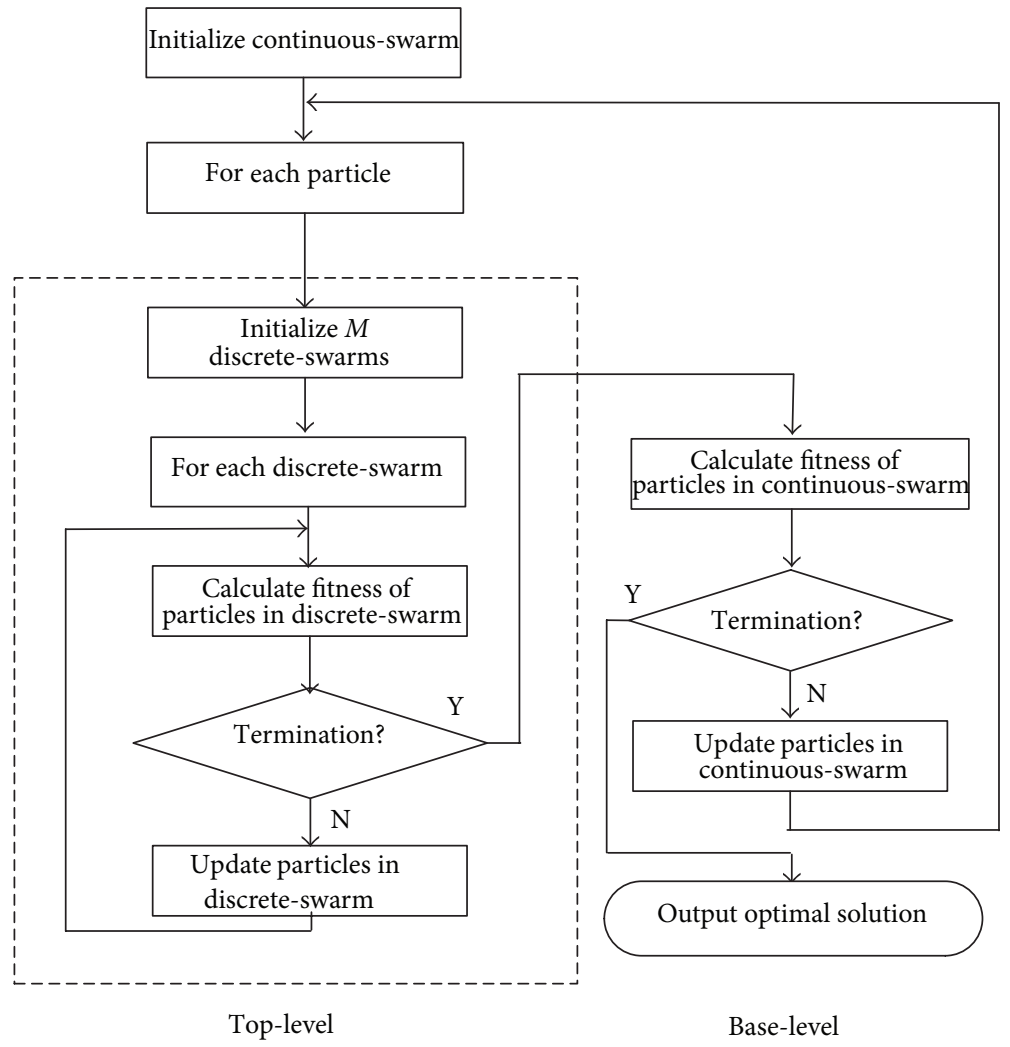

FIgURE 4: The procedures of PSO for the DDM problem.

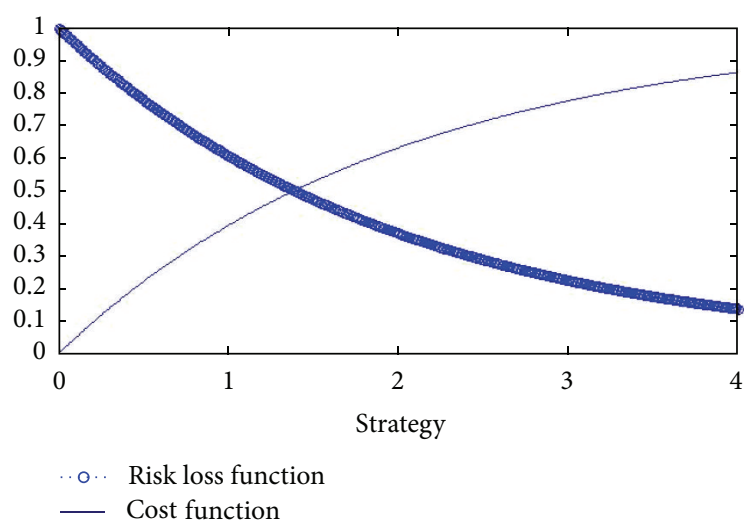

FIGURE 5: The relation between risk loss function and cost function.

Generally, a stronger effect strategy corresponds to a higher cost and a lower risk loss. From the form of the risk loss functions and the cost functions, it can be seen that an additional cost of selecting a stronger effect strategy yields a small reduction on the risk loss, see Figure 5.

For the DDM model with incentives, a bonus $\$ 400$ (bonus $_{i}=400$ ) is taken as an incentive, when the partner reduces his/her risk loss below the target value $\$ 1,000\left(\mathrm{ER}_{i}=1000\right)$ of the owner. The activated cost parameter $\gamma$ is set 0.8 , so the activated cost which the partner
TABLE 1: The values of data.

\begin{tabular}{lc}
\hline Input data & Set values \\
\hline $\begin{array}{l}\text { Number of member in the } \operatorname{VE}(M+1) \\
\text { Upper bound of total budget }\left(B_{\max }\right)\end{array}$ & 2 \\
$\begin{array}{l}\text { Initial risk loss of owner and the partner }\left(L_{i}^{\text {initial }}, 200\right. \\
i=0,1)\end{array}$ & $\$ 3,000$ \\
Number of risk factors for the partner $(N)$ & 10 \\
Number of available risk control strategies for each & 4 \\
risk factor $(W)$ & $\$ 400$ \\
Bonus for the partner $\left(\right.$ bonus $\left._{i}\right)$ & $\$ 1,000$ \\
Target value of risk loss by the owner $\left(\mathrm{ER}_{i}\right)$ & 0.8 \\
Activated cost parameter $(\gamma)$ & $\$ 320$ \\
Activated cost $\left(\right.$ cost $\left._{\mathrm{ac}}\right)$ &
\end{tabular}

will take is $\$ 320$. Table 1 summarizes important input data used in the study.

\subsection{Experimental Results}

4.2.1. Centralized Mechanism. The results of the centralized mechanism are used to be the base case for further improvement. As seen in Table 2, the best computational results of this mechanism can obtain the total VE risk management benefit of $\$ 2,410$; the risk loss of partner is reduced to $\$ 1,286$. Even though there is a causal linkage in the VE where all channels of information sharing can be established, there is 
TABLE 2: Computational results of the centralized mechanism and DDM mechanism with incentives.

\begin{tabular}{lcc}
\hline Performances of the VE & $\begin{array}{c}\text { Centralized } \\
\text { mechanism }\end{array}$ & $\begin{array}{c}\text { DDM } \\
\text { mechanism }\end{array}$ \\
\hline Benefit of VE (\$) & 2,410 & 2,755 \\
Benefit from owner (\$) & 1,296 & 1,493 \\
Benefit from partner $(\$)$ & 1,014 & 1,263 \\
Benefit of partner (\$) & - & 2,347 \\
Investment for owner (\$) & 500 & 596 \\
Investment for partner $(\$)$ & 700 & 604 \\
Total investment $(\$)$ & 1,200 & 1,200 \\
Risk loss of partner $(\$)$ & 1,286 & 733 \\
Cost of partner $(\$)$ & 697 & 870 \\
Strategies combination for & 1003332300 & 0332111000 \\
partner & No & Yes \\
Get bonus from owner & &
\end{tabular}

TABLE 3: Parameters setting of PSO for the two optimization problems.

\begin{tabular}{lc}
\hline Parameters & Value \\
\hline$N_{\text {PC }}$ & 100 \\
$N_{\text {PD }}$ & 80 \\
$N_{\text {IT }}$ & 80 \\
$N_{\text {IB }}$ & 80 \\
$c_{1}$ (continuous problem) & 2.05 \\
$c_{2}$ (continuous problem) & 2.05 \\
$c_{1}$ (discrete problem) & 2 \\
$c_{2}$ (discrete problem) & 2 \\
\hline
\end{tabular}

no financial incentive between either parties. Owner-partner relationships have traditionally been competitive with each other in a winner-loser relationship. Without having benefit sharing, a coordination relationship would be difficult to be established. In this case, the partner does not have a high attitude to select a strong enough risk control strategy to mitigate the risk. This results in the highest risk loss as well as the lowest benefit of the whole VE's risk management.

4.2.2. DDM Mechanism with Incentives. The total risk management benefits of the VE from the DDM mechanism are clearly increased from the base case as shown in Table 2, where the comparison of the risk management benefits of the VE under different perspectives is presented. Characteristics of incentive scheme can be explained in the following manner. The owner offers a bonus to the partner when the risk loss of the partner is reduced to the target value of the owner. Under this type of the incentive, the result reveals that accepting the bonus from the owner is the best policy and yields the maximum risk management benefit of the VE, $\$ 2,755$. The benefit of risk management from the two policies is shown in Figure 6. The risk management benefit of the VE increased $14 \%$ from centralized mechanism to DDM mechanism. In detail, the risk management benefit of the VE is a sum of the benefit from the owner and the

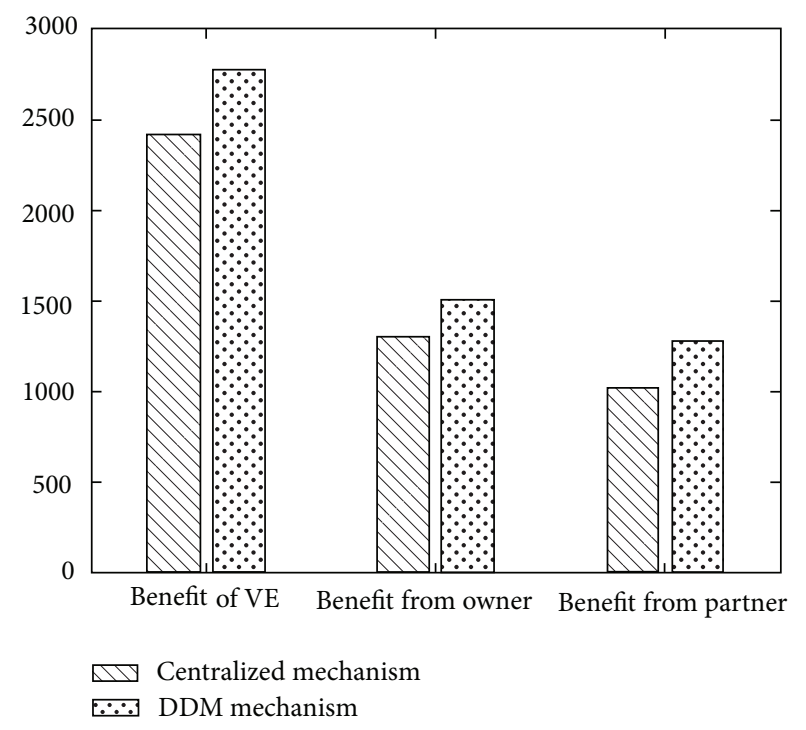

FIGURE 6: Comparison of the risk management benefits from centralized mechanism and DDM mechanism.

partner. More importantly, the benefit from the owner and the partner also increases beyond their benefits from the base case, the increments are $9 \%$ and $22 \%$, respectively. The item "benefits of partner" is only considered in the DDM mechanism, because owner and partner are taken as one in centralized mechanism. Under the centralized mechanism, the risk loss of partner is $\$ 1,286$, but in the DDM mechanism, the risk loss of partner is reduced to $\$ 733$, which is less than the owner's target value $\$ 1000$. It is clear that the winwin game is achieved for both parts in VE under the DDM mechanism.

It would be premature to draw too many conclusions from the initial study. But as a pilot study, the research fulfills its purpose, which is to demonstrate the proposed algorithm by using PSO to compare the traditional centralized mechanism without incentive schemes and the functional DDM mechanism with incentive schemes. Although the solutions given by PSO may not always be optimal, it has proven to generate reasonably good solutions. This is far better than comparing a system with better parameter settings to another system with poor parameter settings. The two optimization problems have the same parameter settings of PSO, which is shown in Table 3.

4.3. Sensitivity Analysis. The results obtained from this study reveal a preliminary outline of benefits from providing incentives among partners in the VE. More experiments of this perspective are still required. This sensitivity of input parameters, as they are usually not known, is another area where further study is needed. Even though there are many variable parameters in the system, not all of them have a major influence on the outcome. Therefore, the incentive scheme (i.e., bonus) is selected to do the sensitivity analysis on the main focus of the study. PSO is used to search for 


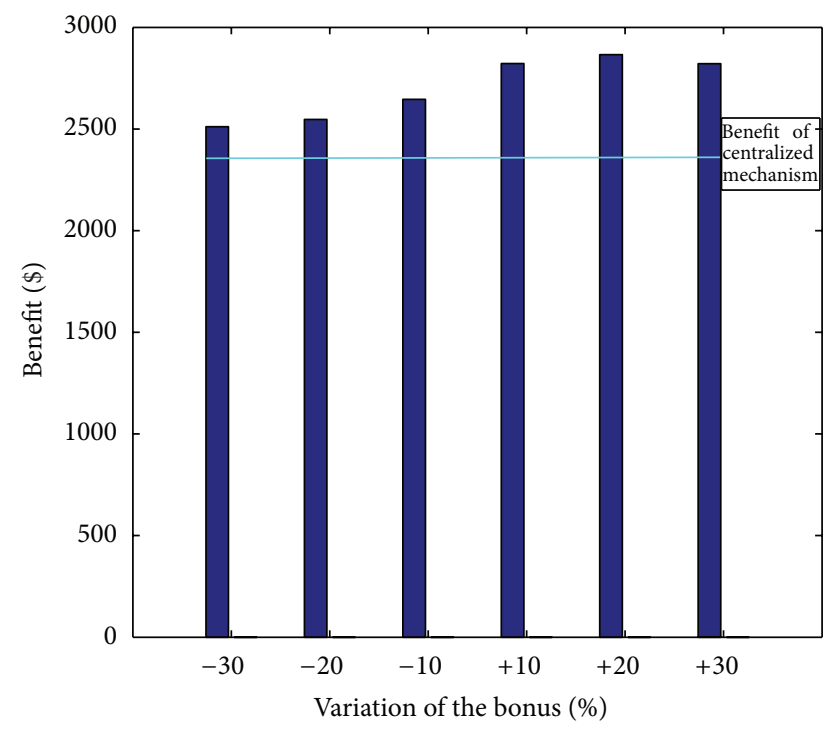

FIGURE 7: Effect of the bonus variation on the performance of VE.

accepting or rejecting the bonus; the amount of bonus in this study is always fixed at $\$ 400$.

By varying the bonus $(-30 \%,-20 \%,-10 \%,+10 \%,+20 \%$, and $+30 \%$ ) with DDM mechanism related to the bonus scheme, Figure 7 illustrates that all of these policies can still maintain their VE benefits beyond what the benefits from the centralized mechanism can offer. Figure 8 shows the variety of partner's benefit under these policies.

When the bonus is increased, the benefits of the owner and the partner are both increased. However, it is noticeable that when the bonus is increased up to $30 \%$, the partners benefit would not increase more than the case of the $20 \%$ increment. Similarly, the owner would not be able to gain the highest benefit under the $30 \%$ increment of the bonus. This is because the objective is set to optimize the benefit of the VE (not benefit of partner), and according to the form of the risk loss functions and the cost functions, an additional cost of selecting a stronger effect strategy yields a small reduction on the risk loss. When the bonus is too high, there is less of a chance that the partner is willing to offer it since it would jeopardize his/her own benefit as well as the benefit of VE. Similarly, when the bonus is too low, there is not enough incentive for the partner to obtain the bonus, which is also difficult to reduce the risk loss to the target value for the partner. As a result, it is back to the base case where the partner will not select strong enough strategies under the fixed allocated budget.

\section{Conclusions}

The strategic partnerships among the members in an FTs supply chain, which is organized as a VE, are essential for improving the performance and responsiveness of the VE. Coordination among the members of VE is a potential way to establish a strategic partnership. However, under conflicting interests among members from different companies,

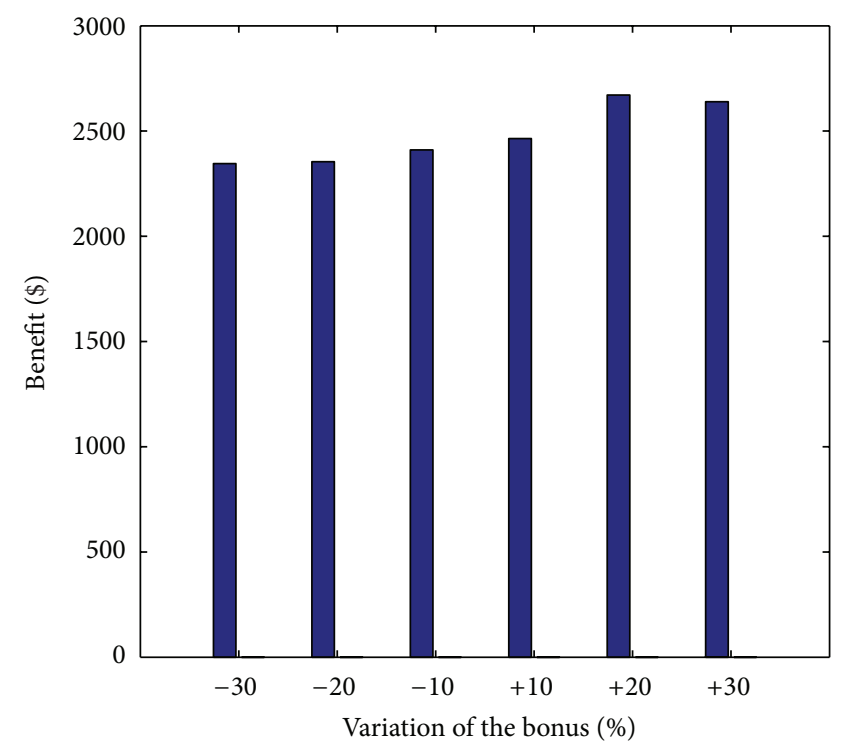

FIgURE 8: Effect of the bonus variation on the performance of partner.

such coordination is not easily implemented. This paper introduced a DDM mechanism with incentive schemes in forming the coordination. The DDM model with incentive schemes has been identified and put into comparison with the centralized one. The simulation results show that appropriate decision mechanism can allow the VE to achieve the best performance (the highest benefit of risk management) and establish a practicable strategic partnership. Besides the FTs supply chain organized as VE, the proposed DDM mechanism is also very useful for other industry supply chains which can be organized as VEs.

There are also several areas for further research. First, it is assumed that all information refers to the idea that risk management process is available to owner. This may not be suitable in some situations where the asymmetric information among the members of VE is possible. Second, the study can be extended to other types of model configuration, such as multiple partners and also different types of coordination: rebate, profit sharing, or other incentive schemes. This would make the study more realistic and cover wider perspectives.

\section{Appendix}

The position of particle $\mathbf{X}_{t}$ is updated by Algorithm 1 .

\section{Acknowledgments}

This work is supported by the National Natural Science Foundation of China under Grant no. 71071028, no. 71021061, no. 70931001, and no. 61070162, the National Science Foundation for Distinguished Young Scholars of China under Grant no. 61225012; the Specialized Research Fund for the Doctoral Program of Higher Education for the Priority Development Areas under Grant no. 20120042130003; Specialized Research 
Fund for the Doctoral Program of Higher Education under Grant no. 20110042110024 and no. 20100042110025; the Specialized Development Fund for the Internet of Things from the Ministry of Industry and Information technology of China; the Fundamental Research Funds for the Central Universities under Grant no. N110204003.

\section{References}

[1] W. H. Davidow and M. S. Malone, The Virtual Corporation: Structuring and Revitalizing the Corporation for the 21st Century, HarperCollins, New York, NY, USA, 1992.

[2] E. Brunelle, "Do virtual enterprises exist? A proposed analysis model," International Journal of E-Business Management, vol. 3, no. 2, pp. 43-55, 2009.

[3] H. Zhi, "Risk management for overseas construction projects," International Journal of Project Management, vol. 13, no. 4, pp. 231-237, 1995.

[4] T. K. Das and B. S. Teng, "Resource and risk management in the strategic alliance making process," Journal of Management, vol. 24, no. 1, pp. 21-42, 1998.

[5] M. Grabowski and K. H. Roberts, "Risk mitigation in virtual organizations," Organization Science, vol. 10, no. 6, pp. 704-721, 1999.

[6] W. H. Ip, M. Huang, K. L. Yung, and D. Wang, "Genetic algorithm solution for a risk-based partner selection problem in a virtual enterprise," Computers and Operations Research, vol. 30, no. 2, pp. 213-231, 2003.

[7] J. Karjalainen, T. Haahtela, P. Malinen, and V. Salminen, "Profit and risk sharing in a virtual enterprise," International Journal of Innovation and Technology Management, vol. 1, no. 1, pp. 75-92, 2004.

[8] M. Huang, W. H. Ip, H. Yang, X. Wang, and H. C. W. Lau, "A fuzzy synthetic evaluation embedded tabu search for risk programming of virtual enterprises," International Journal of Production Economics, vol. 116, no. 1, pp. 104-114, 2008.

[9] Z. Wei, L. Lu, and Z. Yanchun, "Using fuzzy cognitive time maps for modeling and evaluating trust dynamics in the virtual enterprises," Expert Systems with Applications, vol. 35, no. 4, pp. 1583-1592, 2008

[10] T.-Y. Chen, Y.-M. Chen, C.-J. Lin, and P.-Y. Chen, "A fuzzy trust evaluation method for knowledge sharing in virtual enterprises," Computers and Industrial Engineering, vol. 59, no. 4, pp. 853-864, 2010.

[11] M. Huang, F. Q. Lu, W.-K. Ching, and T. K. Siu, "A distributed decision making model for risk management of virtual enterprise," Expert Systems with Applications, vol. 38, no. 10, pp. 13208-13215, 2011.

[12] F. Chen, A. Federgruen, and Y. S. Zheng, "Coordination mechanisms for a distribution system with one supplier and multiple retailers," Management Science, vol. 47, no. 5, pp. 693708, 2001.

[13] N. Chiadamrong and K. Prasertwattana, "A comparative study of supply chain models under the traditional centralized and coordinating policies with incentive schemes," Computers and Industrial Engineering, vol. 50, no. 4, pp. 367-384, 2006.

[14] C. Schneeweiss, "Distributed decision making-a unified approach," European Journal of Operational Research, vol. 150, no. 2, pp. 237-252, 2003.

[15] C. Schneeweiss, Distributed Decision Making, Springer, Berlin, Germany, 2nd edition, 2003.
[16] T. M. Edgington, T. S. Raghu, and A. S. Vinze, "Using process mining to identify coordination patterns in IT service management," Decision Support Systems, vol. 49, no. 2, pp. 175-186, 2010.

[17] F. Valdez, P. Melin, and O. Castillo, "An improved evolutionary method with fuzzy logic for combining particle swarm optimization and genetic algorithms," Applied Soft Computing, vol. 11, no. 2, pp. 2625-2632, 2011.

[18] J. Kennedy and R. Eberhart, "Particle swarm optimization," in Proceedings of the IEEE International Conference on Neural Networks, IV, pp. 1942-1948, IEEE Service Center, Perth, Australia, December 1995.

[19] H. Qi, L. M. Ruan, M. Shi, W. An, and H. P. Tan, "Application of multi-phase particle swarm optimization technique to inverse radiation problem," Journal of Quantitative Spectroscopy and Radiative Transfer, vol. 109, no. 3, pp. 476-493, 2008.

[20] H. S. Yazdi and S. Effati, "Eigenvalue spread criteria in the particle swarm optimization algorithm for solving of constraint parametric problems," Applied Mathematics and Computation, vol. 192, no. 1, pp. 40-50, 2007.

[21] P. Pongchairerks and V. Kachitvichyanukul, "A particle swarm optimization algorithm on job-shop scheduling problems with multi-purpose machines," Asia-Pacific Journal of Operational Research, vol. 26, no. 2, pp. 161-184, 2009.

[22] Y. Marinakis and M. Marinaki, "A hybrid multi-swarm particle swarm optimization algorithm for the probabilistic traveling salesman problem," Computers \& Operations Research, vol. 37, no. 3, pp. 432-442, 2010.

[23] P. Köchel and S. Thiem, "Search for good policies in a singlewarehouse, multi-retailer system by particle swarm optimisation," International Journal of Production Economics, vol. 133, no. 1, pp. 319-325, 2011. 


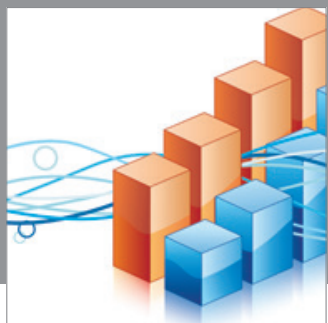

Advances in

Operations Research

mansans

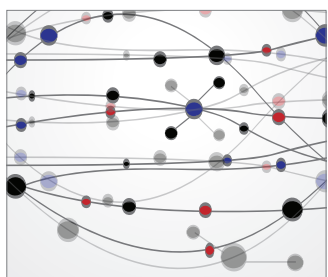

The Scientific World Journal
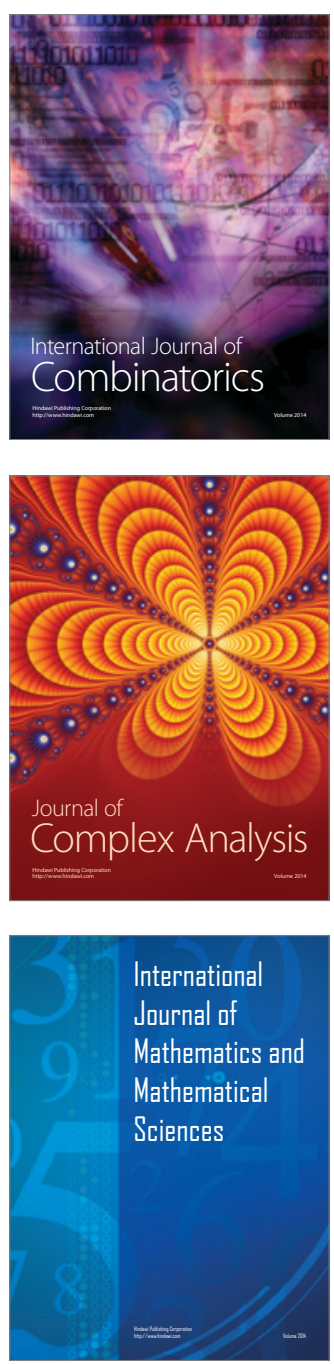
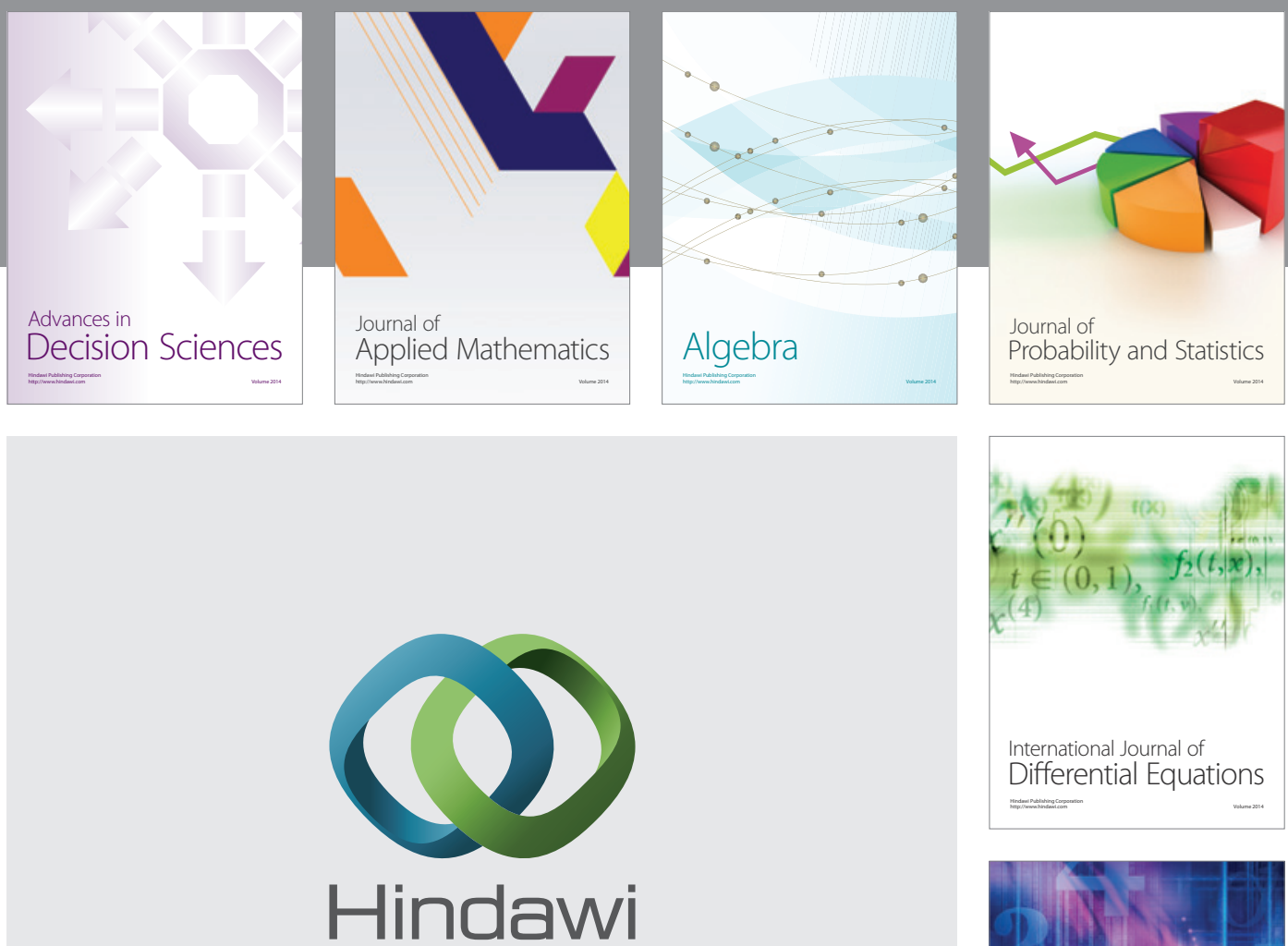

Submit your manuscripts at http://www.hindawi.com
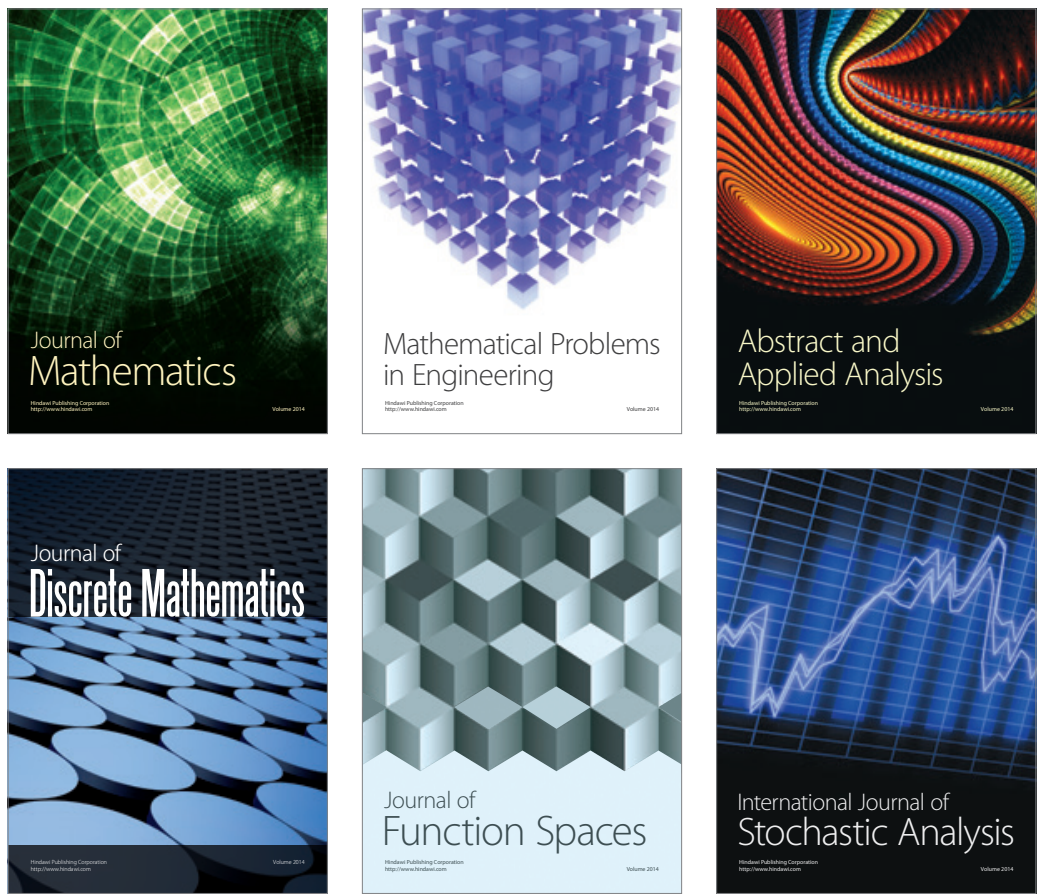

Journal of

Function Spaces

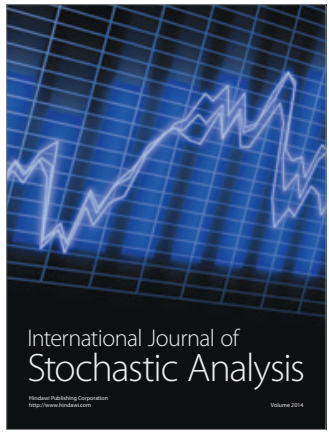

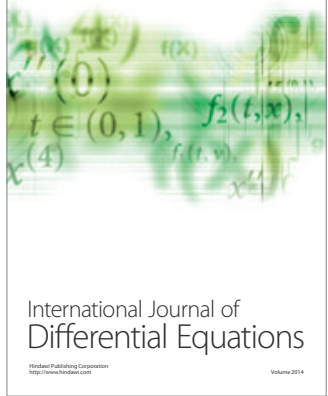
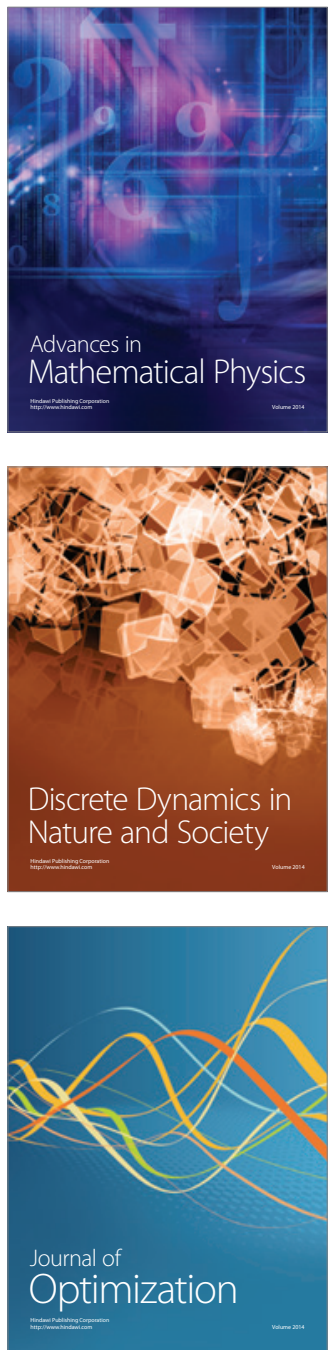